\title{
Pesquisa de Sangue Oculto nas Fezes e Achado Colonoscópico em 60 Pacientes
}

\section{Fecal Occult Blood Test and Colonoscopy Diagnosis in 60 Patients}

\author{
MIRIAM PIRATININGA JATOBÁ ${ }^{1}$; PAULOAZEREDO PASSOS CANDELÁRIA²; WILMAR ARTUR KLUG³; \\ CHIA BINFANG ${ }^{4}$; PERETZCAPELHUCHNIK ${ }^{5}$ \\ ${ }^{1}$ Estagiária da Disciplina; ${ }^{2}$ Mestre em Cirurgia $;{ }^{3}$ Professor Titular; \\ ${ }^{4}$ Professor Adjunto; ${ }^{5}$ Professor Titular
}

\begin{abstract}
JATOBÁ MP; CANDELARIA PAP; KLUG WA; FANG CB; CAPELHUCHNIK P. Pesquisa de Sangue Oculto nas Fezes e Achado Colonoscópico em 60 Pacientes. Rev bras Coloproct, 2008;28(4): 425-430.

RESUMO: Introdução: $O$ carcinoma colo-retal ocorre em alta porcentagem, e é causa comum de morte. A maior incidência se dá nas mais altas faixas etárias, e tanto homens como mulheres são igualmente afetados. Há diferenças no risco para desenvolver carcinoma. Exames preventivos são importantes para reduzir a mortalidade. A pesquisa de sangue oculto nas fezes é um método antigo, mais simples que os demais e se baseia na idéia que os pólipos e os carcinomas sangram intermitentemente, e nos últimos anos houve maior interesse nessa técnica. Objetivo: avaliar as vantagens da pesquisa de sangue oculto nas fezes como método de triagem na detecção de lesões pré-malignas e malignas em nossa população sintomática, correlacionando-a com os resultados da colonoscopia. Método: realizou-se estudo em 60 pacientes da Santa Casa de São Paulo, sintomáticos e com indicação de colonoscopia, que haviam antes realizado pesquisa de sangue oculto nas fezes com o método da benzidina. Resultados: A média das idades foi de 54,2 anos, variando de 12 a 92 anos, sendo 31 mulheres e 28 homens. Os resultados dos exames de sangue oculto foram positivos em 25 e negativos em 34. Várias lesões causadoras de sangramento foram diagnosticadas, e observamos que nos cálculos aritméticos sobre os resultados, a pesquisa do sangue oculto revelou sensibilidade de $63,7 \%$, especificidade de $81 \%$, valor preditivo positivo de $28 \%$ e negativo de $\mathbf{8 8 \%}$. Conclusão: os resultados da aplicação de um método de triagem mostra que a pesquisa do sangue oculto fecal pela benzidina, é exame de eleição para ser aplicado em programas sociais de prevenção de carcinoma coloretal para pacientes sintomáticos.
\end{abstract}

Descritores: Sangue oculto nas fezes, colonoscopia, carcinoma, cólon e reto.

\section{INTRODUÇ̃̃̃O}

O carcinoma colo-retal ocorre em elevada porcentagem e é causa comum de morte. A sobrevivência melhorou nas últimas décadas, houve melhora e avanços terapêuticos e ênfase em programas de prevenção ${ }^{1}$. A maior incidência se dá nas faixas etárias maiores, e tanto homens como mulheres são igualmente afetados. Há diferenças no risco para desenvolver carcinoma. O risco alto corresponde a algumas condições hereditárias como polipose familiar e carcinoma colo-retal hereditário (HNPCC). Por estas razões há diversos métodos de triagem, sendo a pesquisa do sangue oculto nas fezes anual adequado para o risco comum (baixo), ficando a colonoscopia indicada no risco médio e alto.

As tentativas de prevenção interferem na progressão de um pólipo benigno para um carcinoma, que se dá entre 5 e 10 anos ${ }^{1}$. Considerando que o carcinoma colo-retal está relacionado aos pólipos, o diagnóstico precoce deles e sua remoção permite diminuir a incidência do carcinoma ${ }^{2,3}$.

Dentre as técnicas de triagem citam-se o exame de sangue oculto nas fezes, enema opaco,

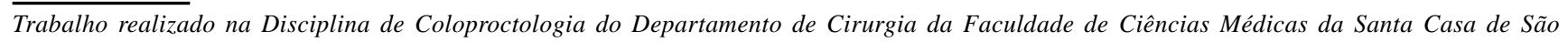
Paulo. 
retossigmoidoscopia flexível e colonoscopia, como também outras alternativas como o teste como DNA e colonoscopia virtual, válidas e recomendadas para pessoas com risco comum (baixo) com idade maior de 50 anos. Já a colonoscopia é o método preferencial para identificar o carcinoma ${ }^{4}$. Ela oferece as vantagens de visualização completa do cólon associadas ao potencial terapêutico. O exame é recomendado depois de resultados positivos obtidos em outros testes de triagem e a remoção de todo pólipo visível resulta em redução de $76 \%$ a $90 \%$ da incidência de carcinoma ${ }^{5}$. Contudo, não há provas que demonstrem a superioridade da colonoscopia sobre outras técnicas de triagem, nem qualquer informação indicando diretamente que um programa periódico de colonoscopia resulte em redução na mortalidade para pessoas com risco comum ${ }^{1}$. Havendo adenomas, estão estabelecidos critérios para a freqüência de novos exames, bem como para acompanhar pacientes após polipectomias ou ressecções por carcinoma. A sensibilidade da colonoscopia convencional para detecção de lesões é de $87,5 \%$, e se feita por profissionais experientes aumenta para mais de $95 \%{ }^{6}$.

Procedimentos de baixo custo e de fácil execução também são utilizados para a detecção precoce do carcinoma. A sigmoidoscopia de fibra ótica está substituindo o instrumento rígido como método de triagem, pois tem melhor custo-benefício como método de triagem quando comparada ao exame de sangue oculto nas fezes e colonoscopia ${ }^{7}$. Entretanto, a sigmoidoscopia e a pesquisa de sangue oculto nas fezes têm resultados similares ${ }^{8}$. Embora o enema opaco possa ser usado teoricamente na triagem de pacientes assintomáticos, a carga de trabalho, os gastos e o desconforto são grandes. Já a colonoscopia virtual mostra um resultado promissor na detecção de pólipos maiores ou iguais a $10 \mathrm{~mm}$, mas a sensibilidade varia de 55 a $94 \%^{9}$.

A pesquisa de sangue oculto nas fezes é um método utilizado desde 1864. É mais simples que os demais e se baseia na idéia de que os pólipos e os carcinomas sangram, e há interesse nessa técnica. Vários estudos mostraram redução da mortalidade por carcinoma ${ }^{2,3,10}$, mas sua limitação se deve à baixa sensibilidade. Em nosso meio um recente estudo concluiu que a pesquisa de sangue oculto é um excelente método de rastreamento para pacientes de baixo risco ${ }^{11}$.

A genética molecular do carcinoma colo-retal proporciona a base para a análise de DNA fecal ${ }^{12,13}$. Estudos prévios usando pesquisa fecal baseado em tes- tes de DNA demonstram sensibilidade de 62 a $91 \%$ para carcinoma, 27 a $82 \%$ para adenomas avançados, com especificidade de 93 a $96 \%{ }^{13,14,15,16,17}$.Entretanto, outros referem que a sigmoidoscopia e o exame de sangue oculto nas fezes não são bons métodos de triagem para a detecção precoce de carcinoma, e recomendam colonoscopia ${ }^{18}$.

Em nosso meio há parasitoses intestinais que podem dar resultado falso positivo, mas independente disso é indiscutível o avanço realizado no sentido de tornar viável a pesquisa de sangue oculto nas fezes como método inicial principal de triagem para pesquisa de lesões pré-malignas e malignas 1,2,3,5,7,10,19,20,21, 22,23,25

Com base nestes estudos, o objetivo do trabalho é avaliar as vantagens da pesquisa de sangue oculto nas fezes como método de triagem na detecção de lesões pré-malignas e malignas em nossa população sintomática, correlacionando com os resultados da colonoscopia.

\section{CASUÍSTICA E MÉTODO}

Realizou-se estudo prospectivo na Disciplina de Coloproctologia do Departamento de Cirurgia da Santa Casa de São Paulo, já avaliado pela Comissão Científica do Departamento de Cirurgia e pelo Comitê de Ética em Pesquisa.

Por ocasião do $\mathrm{I}^{\circ}$ Curso Prático de Colonoscopia e $\mathrm{I}^{\circ}$ Mutirão de Colonoscopia da Santa Casa de São Paulo foram selecionados 60 pacientes para colonoscopia, indicados na rotina, após fazerem pesquisa de sangue oculto. Destes, uma mulher de 81 anos, com sangue oculto negativo, foi excluída por intensa dor abdominal. Os 59 restantes realizaram os dois exames, sangue oculto e colonoscopia. A média das idades foi de 54,2 anos, de 22 a 92 anos, sendo $31 \mathrm{mu}-$ lheres e 28 homens. Os resultados dos exames de sangue oculto foram positivos em 25 e negativos em 34 .

Os pacientes eram sintomáticos e as queixas sugeriram doenças do cólon, ocorrendo: alteração do hábito intestinal (20), dores abdominais (17), sangramento (14), constipação (13), perda ponderal (12), diarréia (10) e muco nas fezes (5). A indicação dos exames de colonoscopia foi baseada em critérios da Disciplina de Coloproctologia (Tabela 1)

O método empregado para a pesquisa de sangue oculto foi a reação da benzidina, com dieta especial de três dias precedendo o exame. Para identificar san- 
Tabela 1 - Indicação de colonoscopia correlacionado com o achado de sangue oculto.

\begin{tabular}{lccc}
\hline Indicações & Pacientes & Sangue oculto positivo & Sangue oculto negativo \\
\hline Alteração do hábito intestinal & 20 & 7 & 13 \\
Controle pós-operatório & 18 & 11 & 7 \\
Sangramento nas fezes & 16 & 5 & 11 \\
Emagrecimento & 9 & 5 & 4 \\
Dor abdominal & 9 & 4 & 5 \\
Anemia & 3 & 1 & 2 \\
Outros & 18 & 7 & 11 \\
\hline
\end{tabular}

gue oculto nas fezes é imperiosa a reação entre a peroxidase ou pseudoperoxidase com o eritrócito contendo a hemoglobina, que adicionado ao peróxido de hidrogênio faz o teste. Da mesma forma que o guáiaco e a ortotoluidina, a benzidina é um indicador que é oxidado a um composto azul. Sua avaliação é subjetiva, com resultados semelhantes ao do guáiaco.

Para análise estatística dos resultados foi empregado o Teste do $\mathrm{X}^{2}$ e Teste Exato de Ficher. Foram testados sensibilidade, especificidade, valor preditivo positivo e negativo.

\section{RESULTADOS}

Os resultados foram expressos consoante a presença ou ausência de sangue oculto nas variadas condições clínicas testadas. Nas tabelas 2,3,4 e 5 estão as relações entre os achados de colonoscopia de portadores de hemorróidas e o sangue oculto, achados colonoscópicos e sua relação com o teste de sangue oculto, e resultados dos exames anatomopatológicos das lesões ressecadas.
O paciente com diagnóstico de adenoma tubular com displasia de alto grau foi submetido à ressecção do cólon, encontrando-se adenocarcinoma moderadamente diferenciado. $\mathrm{O}$ diagnóstico de sangue oculto foi positivo em $100 \%$ dos pacientes com carcinoma.

Em relação aos cálculos aritméticos sobre os resultados, observamos que o método de pesquisa do sangue oculto, nesta casuística, revelou sensibilidade de $63,7 \%$, especificidade de $81 \%$, valor preditivo positivo de $28 \%$ e negativo de $88 \%$.

\section{DISCUSSÃO}

A necessidade de um método de triagem para busca ativa do carcinoma colo-retal não é mais motivo de discussão. No Brasil, o melhor método é aquele que reúna preço baixo, fácil execução, sensibilidade elevada e aceitação social. Trabalhando com os recursos existentes o exame de sangue oculto é alternativa atraente apesar de não preencher todos os requisitos.

Tabela 2 - Relação entre o achado da colonoscopia de pacientes portadores de hemorróidas e o exame de sangue oculto.

\begin{tabular}{lccc}
\hline Colonoscopia & $\begin{array}{c}\text { Pacientes portadores } \\
\text { de hemorróidas }\end{array}$ & Sangue oculto positivo & Sangue oculto negativo \\
\hline Pólipos & 3 & 1 & 2 \\
Divertículos & 1 & 0 & 1 \\
Ectasia vascular & 1 & 1 & 0 \\
Inflamação & 1 & 1 & 0 \\
Normal & 1 & 1 & 0 \\
TOTAL & 7 & 4 & 3 \\
\hline
\end{tabular}


Miriam Piratininga Jatobá e Cols.

Tabela 3 - Correlação entre o achado da colonoscopia e o exame de sangue oculto.

\begin{tabular}{lccc}
\hline Colonoscopia & Pacientes & Sangue oculto positivo & Sangue oculto negativo \\
\hline Pólipos & 16 & 8 & 8 \\
Divertículos & 14 & 1 & 13 \\
Inflamação & 13 & 6 & 7 \\
Alterações vasculares & 2 & 2 & 0 \\
Tumor & 2 & 2 & 0 \\
Normal & 12 & 6 & 6 \\
TOTAL & 59 & 25 & 34 \\
\hline
\end{tabular}

Tabela 4 - Resultado anatomopatológico dos pólipos ressecados.

\begin{tabular}{lccc}
\hline Anátomo-patológico & Pacientes & Sangue oculto positivo & Sangue oculto negativo \\
\hline Pólipo adenomatoso & 9 & 5 & 4 \\
Pólipo inflamatório & 5 & 2 & 3 \\
Pólipo hiperplásico & 2 & 1 & 1 \\
TOTAL & 16 & 8 & 8 \\
\hline
\end{tabular}

Vários são os métodos para pesquisa de sangue oculto nas fezes. Entre eles destacamos o imunoquímico, o guáiaco - utilizado desde 1864-, e o da benzidina, que empregamos. Nossos pacientes eram sintomáticos e tinham indicação para colonoscopia, mas poderia divergir o resultado se os pacientes não o fossem. A escolha do método da benzidina não é compartilhada por outros, mas observamos resultado semelhante àqueles ${ }^{20,22}$.

A não realização de exames parasitológicos de fezes pode interferir nos resultados. Porém, nos 25 doentes que apresentaram exame de sangue oculto positivo, em 17 havia doenças que justificaram o sangramento e, portanto a presença de parasitas não determinou influência significativa (p>0,05) (Tabela 3 ).
Viu-se que sete pacientes eram portadores de hemorróidas. Como sua principal manifestação é sangramento anal haveria interferência nos resultados.

Porém, somente um com hemorróidas apresentou sangue oculto positivo e o exame colonoscópico foi normal $(p=0,532)$. Assim, vimos que a presença de hemorróidas com sangue oculto positivo não interferiu na pesquisa de lesão pré-maligna ou maligna, podendo participar do protocolo de triagem (Tabela 2).

No exame anatomopatológico dos pólipos ressecados e dos tumores dos pacientes com sangue oculto positivo, observamos que sete (28\%) apresentaram diagnóstico de lesão pré-maligna ou maligna. Estratificandoos encontramos cinco (20\%) com lesão pré-maligna (adenoma) e dois (8\%) com lesão maligna. Estes resultados são semelhantes a outros da literatura ${ }^{22}$.

Tabela 5 - Resultado anatomopatológico dos tumores por biópsia.

\begin{tabular}{lccc}
\hline Anátomo-patológico & Pacientes & Sangue oculto positivo & Sangue oculto negativo \\
\hline Adenoma tubular & 1 & 1 & 0 \\
com displasia de alto grau & & & 0 \\
Adenocarcinoma mucoso & 1 & 1 & 0 \\
TOTAL & 2 & 2 & \\
\hline
\end{tabular}


Para que um método de triagem seja efetivo, é preciso que tenha alta sensibilidade. Observamos que no exame de sangue oculto ela é de $63,7 \%$. Contrariamente, a especificidade foi $81 \%$, não tão efetiva quando se trata de métodos de triagem populacional. $\mathrm{O}$ valor preditivo positivo foi de $28 \%$. O valor preditivo negativo foi de $88 \%$, configurando que quando o paciente apresenta um resultado negativo, implica ser altamente provável que não tenha lesão pré-maligna ou maligna. Nossos valores são similares aos da literatura, onde a especificidade da pesquisa de sangue oculto nas fezes, utilizando o Haemocult para adenomas ou carcinomas foi de $85,9 \%$ e o valor preditivo positivo foi de $27,5 \%{ }^{23}$.

O exame de sangue oculto com resultado falso negativo é motivo de discussão, e por este motivo fora abandonado, mas recentemente revivido. Para reduzir o número de resultados falso-negativos, aconselha-se a realização de seis esfregaços de Haemoccult de cada indivíduo, representado por duas porções separadas de três defecações consecutivas, regime amplamente aceito ${ }^{24}$. Em nosso estudo o resultado falso negativo foi elevado, e isto se deve ao fato de que o método de colheita se restringiu a uma única amostra de fezes. Com o aumento do número este problema deverá ser solucionado ${ }^{24}$.

Vários foram os pacientes sintomáticos com exame de sangue oculto positivo, mas não foi possível predizer a existência de carcinoma colo-retal senão através da colonoscopia. Isto também é comparável à literatura ${ }^{26}$.

Considerando os resultados apresentados e a necessidade de um método de triagem, acreditamos que a pesquisa do sangue oculto deva ser o exame de eleição para ser aplicado em programas sociais de prevenção de carcinoma colo-retal, para pacientes sintomáticos.

\section{CONCLUSÃO}

No estudo pudemos concluir que o método de pesquisa de sangue oculto ainda é alternativa na investigação inicial e de baixo custo para pesquisa de carcinoma colo-retal.

\begin{abstract}
Background: colon and rectal carcinoma are frequent occurrences and common causes of death. The elderly, both male and female, are the most affected group and preventive exams are essential to reduce mortality rates. The fecal occult blood tests are long-established and simple methods which are based on the idea that polyps and carcinomas bleed frequently. In recent times, the interest in these methods grew considerably. Objective: the study evaluates the relevance of fecal occult blood test as a screening method in symptomatic patients compared to colonoscopy results. Method: 60 symptomatic patients with colon and rectal ailments at Santa Casa of São Paulo Hospital underwent fecal occult blood tests with benzidine, followed by colonoscopic exam. Results: the average age was 54.2, being 31 of them women and 28 men. The fecal occult blood test was positive in 25 and negative in 34. Lesions were identified as the cause of bleeding and the fecal occult blood test had a sensibility of $63.7 \%$, specificity of $81 \%$, predictive positive value of $28 \%$ and negative of $88 \%$. Conclusion: the present results show that the fecal occult blood test should be applied in social programs of carcinoma prevention in symptomatic patients.
\end{abstract}

Keywords: Occult blood test, colonoscopy, carcinoma, colon and rectum.

\section{REFERÊNCIAS}

1. Bromer MQ; Weinberg DS. Screening for Colorectal Cancer Now and the Near Future. Semin Oncol, 2005; 32:3-10.

2. Hardcastle JD; Chamberlain JO; Robinson MH; Moss SM; Amar SS; Balfour TW et al. Randomised controlled trial of faecal-occult-blood screening for colorectal cancer. Lancet, 1996; 348:1472-1477.

3. Kronborg O; Fenger C; Olsen J; Jorgensen OD; Sandergaard O. Randomised study of screening for colorectal cancer with faecal-occult-blood test. Lancet, 1996; 348:1467-1471.
4. Sung JJ; Chan FK; Leung WK; Wu JC; Lau JY; Ching J; To KF; Lee YT; Luk YW; Kung NN; Kwok SP; Li MK; Chung SC. Screening for colorectal cancer in Chinese: comparison of fecal occult blood test, flexible sigmoidoscopy, and colonoscopy. Gastroenterology, 2003; 124(3):608-614.

5. Winawer SJ; Zauber AG; Ho MN et al. Prevention of colorectal cancer by colonoscopic polypectomy. The National Polyp Study Workgroup. N Engl J Med, 1993; 329:1977-1981.

6. Rockey, DC. Virtual Colonoscopy to Screen for Colorectal Cancer. New England Journal of Medicine, 2004; 350(11):11481150. 
7. O'Leary BA; Olynyk JK; Neville AM; Platell CF. Costeffectiveness of colorectal cancer screening: comparison of community-based flexible sigmoidoscopy with fecal occult blood testing and colonoscopy. J Gastroenterol Hepatol, 2004; 19(1):38-47.

8. Segnan N; Senore C; Andreoni B; Arrigoni A; Bisanti L; Cardelli A; Castiglione G; Crosta C; Di Placido R; Ferrari A; Ferraris R; Ferrero F; Fracchia M; Gasperoni S; Malfitana G; Recchia S; Risio M; Rizzetto M; Saracco G; Spandre M; Turco D; Turco P; Zappa M. Randomized trial of different screening strategies for colorectal cancer: patient response and detection rates. J Natl Cancer Inst, 2005; 97(5):347-357 .

9. Florie J; van Gelder RE; Stoker J. Colonography by computed tomography. Eur J Gastroenterol Hepatol, 2005; 17(8):809813.

10. Mandel JS; Bond JH; Church TR; Snover DC; Bradley GM; Schuman LM et al. Reducing mortality from colorectal cancer by screening for fecal occult blood. N Engl J Méd, 1993; 28:1365-1371.

11. Altenburg FL; Biondo-Simões MLP; Santiago A. Pesquisa de sangue oculto nas fezes e correlação com alterações nas colonoscopias. Rev bras Coloproct, 2007; 27 (3): 304-309.

12. Sidransky D; Tokino T; Hamilton SR; et al. Identification of ras oncogene mutations in the stool of patients with curable colorectal tumors. Science, 1992; 256:102-105.

13. Ahlquist DA; Skoletsky JE; Boynton KA,; et al. Colorectal cancer screening by detection of altered human DNA in stool: feasibility of a multitarget assay panel. Gastroenterology, 2000; 119:1219-1227.

14. Dong SM; Traverso G; Johnson C et al. Detecting colorectal cancer in stool with the use of multiple genetic targets. J Natl Cancer Ins, 2001; 93:858-865.

15. Syngal S; Chung D; Willet C et al. The loss of stool DNA mutation abnormalities in colorectal neoplasia after treatment. Gastroenterology, 2003; 124:Suppl 1:A-5.

16. Tagore KS; Lawson MJ; Yucaitis JA et al. Sensitivity and specificity of a stool DNA multitarget assay panel for the detection of advanced colorectal neoplasia. Clin Colorectal Cancer, 2003; 3:47-53.

17. Traverso G; Shuber A; Levin B et al. Detection of APC mutations in fecal DNA from patients with colorectal tumors. N Engl J Med, 2002; 346:311-320.
18. Cheng TI; Wong JM; Hong CF et al. Colorectal cancer screening in asymptomaic adults: comparison of colonoscopy, sigmoidoscopy and fecal occult blood tests. J Formos Med Assoc (China Republic), 2002; 101:685-690.

19. Mandel JS; Bond JH; Bradley M; Snover DC; Church TR; Williams S; Watt G; Schuman LM; Ederer F; Gilbertsen V. Sensitivity, specificity, and positive predictivity of the Hemoccult test in screening for colorectal cancers. Gastroenterology, 1989; 97:597-600.

20. Ko CW; Dominitz JA; Nguyen TD. Fecal occult blood testing in a general medical clinic: comparison between guaiac-based and immunochemical-based tests. Am J Med , 2003; 115(2):111-1114.

21. Veysey M; Leiyten W; Walsh P; Vigenser B. At what age should faecal occult blood screening be undertaken?: 29. J Gastroenterology \& Hepatology, 2005; 20:A16.

22. Morikawa T; Kato J; Yamaji Y; Wada R; Mitsushima T; Shiratori Y. A comparison of the immunochemical fecal occult blood test and total colonoscopy in the asymptomatic population. Gastroenterology, 2005; 129(2):422-428.

23. MacRae FA; Slattery MA; Brown GJ; O’Dwyer MA; Murphy CG; Hibbert ME. Sensitivity of faecal occult blood testing (FOBT) for asymptomatic colorectal cancer and advanced adenomas over a 25-year experience in colorectal cancer screening: 66. J Gastroenterology \& Hepatology, 2005; 20.

24. Greegor DH. Diagnosis of large bowel câncer in the asyntomatic patient. J Amer Med Ass, 1967; 201:943-947.

25. INCA-Instituto Nacional do Câncer - http://www.inca.gov.br/ conteudo_view.asp?id=325, 2005.

26.Ahmed S; Leslie A; Thaha MA; Carey FA; Steele RJ. Lower gastrointestinal symptoms are not predictive of colorectal neoplasia in a faecal occult blood screen-positive population. Br J Surg, 2005; 92(4):478-481.

\section{Endereço para correspondência:}

DR. WILMAR ARTUR KLUG

Alameda Ribeirão Preto, 487 - Ap. 103

01331-001 - São Paulo - SP 